\title{
The Research of Gas Detection Based on data Fusion
}

\author{
Haiqun Wang ${ }^{1, a}$, Dongyun Liü,b \\ ${ }^{1}$ College of Electrical Engineering Hebei United University, Tangshan, China \\ aWanghq0604@yeah.net, b946147894@qq.com
}

Keywords: data fusion, gas detection, STM32F103, upper computer

\begin{abstract}
Aiming at the problem of gas pollution in the environment, the traditional testing equipments often appear the phenomenon of misstatement and false positives. A real-time online monitoring system is designed based on the detecting and control of harmful gases. The lower computer system make the STM32F103 microcontroller as the core, PC as the monitoring center, combining the technology of multi-sensor data fusion, using self-adapting weighting data fusion algorithm and decision level data fusion algorithm based on fuzzy set theory. Through the upper machine people can not only understand the quality of the indoor environment in time, and the system can inhibit the drift and noise of sensors to some extent, improve the measuring accuracy of the monitoring system for harmful gas in the environment, improve system reliability, reduce system fuzziness, enhance the reliability of system and assure the system having good robustness.
\end{abstract}

\section{Introduction}

Due to the restriction of economic development level, at present, the development of our country environment monitoring technology is not mature. Monitoring equipment and technology is relatively backward and the single, low degree of automation, most of gas monitor mainly is field sampling and laboratory analysis instrument as the main technical means, this method is not only low efficiency, high cost, but also costs a lot of manpower and material resources, financial resources, and analysis is slow, cannot satisfy the needs of real-time analysis of gas environmental quality [1]. Even though the market appears on a lot of portable gas detector at present, but the single test species, poor anti-interference ability and the poor precision, which can't satisfy to detect the gas concentration accurately and automatically. Therefore, developing the harmful gas detection system combining data fusion technology which is integrating data acquisition and environmental monitoring has important significance.

\section{Working Principle of System}

The system consists of two parts, the first part is upper computer data processing and display system composed of monitoring software of the computer, the second part is based on single chip microcomputer and sensors as the core, and intelligent detection and control system composed of other peripheral equipment. PC can monitor the concentration of harmful gases as well as the operation condition at any time, which can divide environmental quality into some kinds of grades respectively, each as excellent, good, bad and worse through the groups of data fusion. When the level of harmful gases is poor, PC warns MCU red lights flashing, when the level of harmful gases is worse, the system opens the sound and light alarm circuit, reminding people of harmful gas concentration is too high. 
Three sensors of the same type is used in this system to analyze. First of all, the STM32 uploads the data of gas concentration to the PC through the RS-485 which are detected by the sensors, upload number for five times, after reaching five times, the upper computer uses adaptive weighted data fusion to process data. Then the upper computer uses decision level data fusion based on fuzzy set theory to grade the gas quality according the processed fusion results. Finally, the analysis results will be shown by monitoring the interface and make corresponding alarm response according to the results. The main program flow chart of the system is shown in figure 1 .

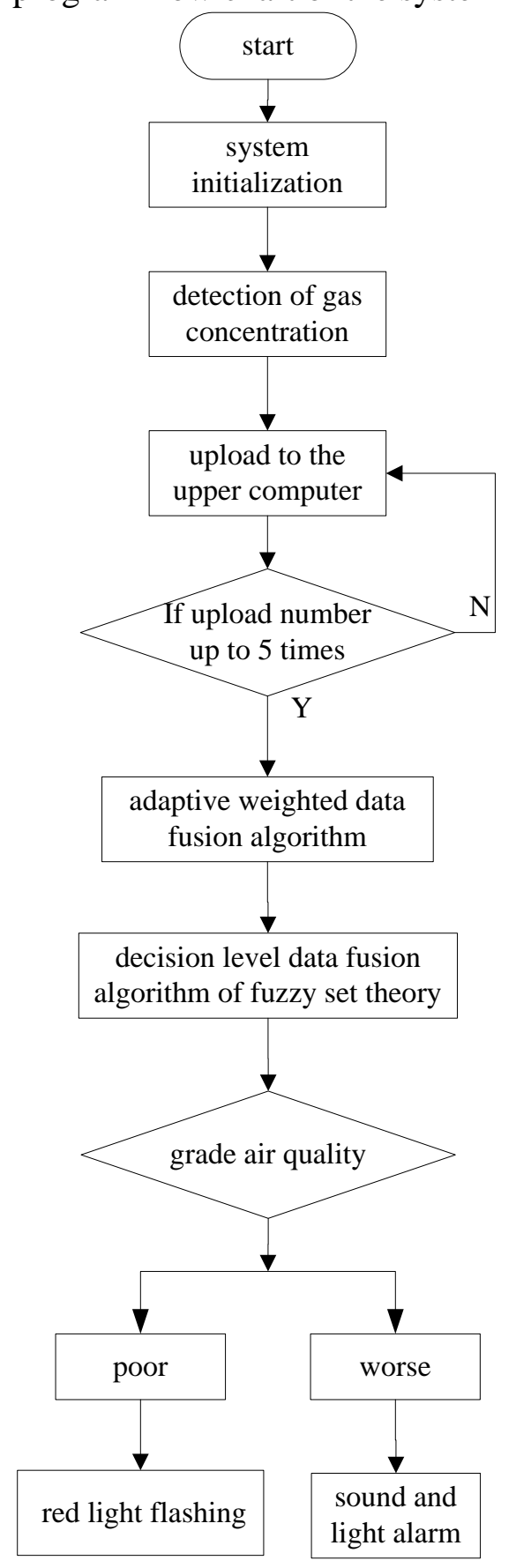

Fig.1The main program flow chart of system

\section{The Design of System}

The hardware circuit of the system is based the single chip processor (STM32) and gas sensors as the core, the peripheral equipment including alarm circuit, sensor 
interface circuit, power circuit, the RS-485 interface circuit, etc., which shoulders the detection and transmission of the gas concentration and other functions for example alarm. The main role of upper computer is data processing and information display through the monitoring interface, and according to the results controlling the MCU to make corresponding response by the RS-485. The structure diagram of the system is shown in figure 2.

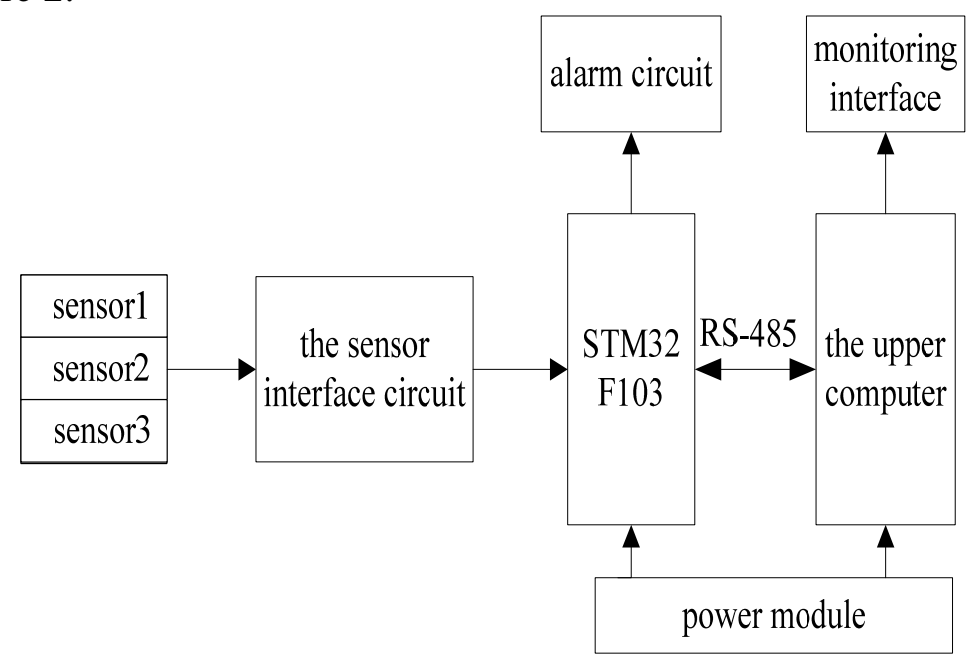

Fig.2 System block diagram

\section{The Data Fusion}

Adaptive Weighted Data Fusion. On the basis of the hardware, the sensor's raw data, using multi-sensors adaptive weighted data fusion algorithm, makes the mean square error and measuring precision for information fusion and accurately figures out. Its advantage is that it does not require any prior knowledge of measurement data, and only on the basis of measurement precision of the measurement data to determine the corresponding weighting of different data and calculate the minimum mean square error value. It can inhibit the drift and noise of sensors to some extent, improve the accuracy of measurement [2]. The model is shown in figure 3.

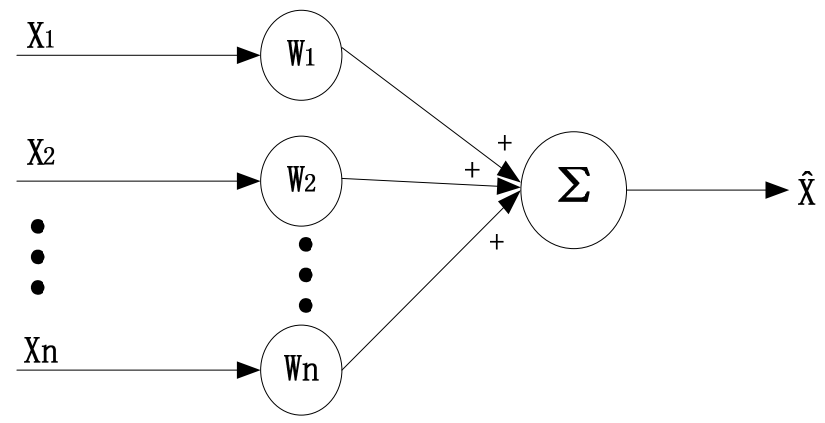

Fig.3 Model of adaptive weighted data fusion

Each sensor in the system all have their corresponding weights $W_{i}$, and $W_{i}$ depends on detection error value of the sensor. If the error value of the test result is small, $W_{i}$ is big, on the contrary $W_{i}$ is small. With minimum variance as a prerequisite, each testing data according to the principle of adaptive calculate the corresponding values, make the results more close to the real value through data fusion [3].

The algorithm of computing process is as follows.

i) Calculate the average of the continuous acquisition data of each sensor $\bar{X}_{i}$. 
ii) Calculate the variance of each sensor $\sigma_{i}^{2}$.

iii) To find the optimal weighted factor $W_{i}$.

iv) To calculate the fusion estimate value $\hat{X}$.

The optimal value and weights of the fused should have the following features.

$$
\begin{gathered}
\sum_{i=1}^{n} W_{i}=1 \\
\hat{X}=\left[\begin{array}{llll}
W_{1} & W_{2} & \cdots & W_{\mathrm{n}}
\end{array}\right]=\left[\begin{array}{llll}
X_{1} & X_{2} & \cdots & X_{\mathrm{n}}
\end{array}\right]^{T}=\sum_{i=1}^{n} W_{i} X_{i}
\end{gathered}
$$

Respectively using arithmetic average algorithm and adaptive weighted data fusion algorithm for data processing, and then respectively comparing with the real value. The system is carbon monoxide as example. The results are shown in the table 1 .

Table10 Fusion value comparison

\begin{tabular}{ccc}
\hline real value $(\mathrm{ppm})$ & arithmetic average value & adaptive weighted value \\
\hline 0.50 & 0.45 & 0.47 \\
1.00 & 1.06 & 1.03 \\
2.00 & 2.05 & 2.02 \\
5.00 & 5.07 & 5.02 \\
10.00 & 10.04 & 10.01 \\
\hline
\end{tabular}

Through contrast the data in the table above, the results of using adaptive weighted data fusion are closer to their true value, and the fusion value of using the arithmetic average algorithm compare with the real value, the deflection is bigger. So the adaptive weighted data fusion algorithm can make full use of the sensor of the original data to achieve the optimum fusion value, which can improve the accuracy of the gas detection.

The Decision Level Data Fusion Based on the Theory of Fuzzy. The results though adaptive weighted data fusion algorithm, combined the weight vector A of the decision level data fusion algorithm based on the theory of fuzzy sets. It is concluded that the quality of the environment. In this system, the determination of index weight of four sets of data(temperature, humidity, carbon monoxide, sulphur dioxide), mainly refer to the environment quality index questionnaires of the relevant experts, combined with 1-9 scaling method [4], using the eigenvector method is the biggest characteristic of matrix $\mathrm{P}$ eigenvectors corresponding to the root, the eigenvector being after normalization and consistency to get the weight of each factor [5].

$$
A=\left[\begin{array}{c}
\frac{2.233}{2.233+1.055+0.448+0.228} \\
\frac{1.055}{2.233+1.055+0.448+0.228} \\
\frac{0.448}{2.233+1.055+0.448+0.228} \\
\frac{0.228}{2.233+1.055+0.448+0.228}
\end{array}\right]=\left[\begin{array}{l}
0.558 \\
0.263 \\
0.122 \\
0.057
\end{array}\right]
$$

Using random consistency index $C R$ to measure the consistency of judge matrix.

$$
C R=\frac{\lambda_{\max }-n}{R I(n-1)}
$$


In the type, $\lambda_{\max }$ is the biggest characteristic root of matrix $\mathrm{P}$ and $\mathrm{n}$ is order of $P . R I$ is random parameter values, through a large number of random calculated, being associated with the matrix order. Used to think that as long as the $C R$ is less than 0.10 , the judgment matrix $P$ has the satisfactory consistency, otherwise have to readjust the P element value[6]. By the calculation:

$$
C R=\frac{4.118-4}{0.89(4-1)}=0.044<0.10
$$

So the judgment matrix consistency is good, $A$ can be used as index weight.

\section{Control GUI}

Visual Basic is a set of development system based on Windows environment, which has the characteristics of simple, easy and efficient quickly occupied the dominant position of the market. It becomes many developers the preferred development tools [7]. Therefore, the system uses Visual Basic 6.0 to develop the monitoring interface of the upper computer which main receives the data of sensor collecting. Then the results will be processed for display with the data fusion algorithm [8].

\section{Conclusions}

This system applies the technology of the sensor, microcontroller technology, data fusion technology and communication technology, implements real-time online detection of the concentration of harmful gas, and the collected data will be transferred the upper computer through the RS-485 and provide certain reference for the people. It is of great significance for gas detection in the environment, people can be the real-time understanding of harmful gases and system operation condition according to the testing results, avoid excess gas produced harm to human body. The system can greatly improved the accuracy of gas detection, make environment of people's life more realistic and credible and reduce the phenomenon of misstatement and false positives.

\section{References}

[1] Wang Xu-Hui. The analysis of optimization of regional human capital allocation in China. Academic Exchange, (4):72-75,2011.

[2] Yan L Y, Liu B S, Zhou D H. The modeling and estimation of asynchronous multirate multisensory dynamic systems. Aerospace Science and Technology, (10):63-71, 2006.

[3] Wen Wei-Min, Wang Mei, Zou Rong, etc. The indoor harmful gas monitoring system based on adaptive weighted data fusion. Journal of Shenyang instrument institute, 2007, 8.

[4] Chen Y M, Huang H C. Fuzzy logic approach to multisensory data association[J]. Mathematics and computers in Simulation, 52(5-6):399-412, 2000.

[5] V.V. SAKHARE, R.V. RALEGAONKAR. Review indoor environmental quality: review of parameters and assessment models. Architectural Science Review, 57(2), 2014.

[6] Jing Li, Yuan Xiao-Hong, Zhang Jie. Command automation system effectiveness evaluation of quantitative scale study China automation society the 21st youth academic conference, 2006, 8. 
[7] Dong Wen-Jun, Jing Jing, Zhang Jing. GPS data processing module design based on VB. Journal of capital normal university, (5): 81-84, 2008.

[8] Li Xiang-Jiang, Peng Jian. The Design of Real-time Data Acquisition Program Based On VB. The Microcomputer Information, 19(10):38-39, 2003. 